\title{
Randomizing quantum states: Constructions and applications
}

\author{
Patrick Hayden, ${ }^{1,2, *}$ Debbie Leung, ${ }^{1,2, \oplus}$ Peter W. Shor, ${ }^{3,}$, \\ ${ }^{1}$ Institute for Quantum Information, Caltech 107-81, Pasadena, CA 91125, USA \\ ${ }^{2}$ Mathematical Sciences Research Institute, 1000 Centennial Drive, Berkeley, CA 94720, USA \\ ${ }^{3} A T$ \& T Labs Research, Florham Park, NJ 07922, USA \\ ${ }^{4}$ Department of Computer Science, University of Bristol, \\ Merchant Venturers Building, Woodland Road, Bristol BS8 1UB, United Kingdom
}

(Dated: May 30, 2006)

\begin{abstract}
The construction of a perfectly secure private quantum channel in dimension $d$ is known to require $2 \log d$ shared random key bits between the sender and receiver. We show that if only near-perfect security is required, the size of the key can be reduced by a factor of two. More specifically, we show that there exists a set of roughly $d \log d$ unitary operators whose average effect on every input pure state is almost perfectly randomizing, as compared to the $d^{2}$ operators required to randomize perfectly. Aside from the private quantum channel, variations of this construction can be applied to many other tasks in quantum information processing. We show, for instance, that it can be used to construct LOCC data hiding schemes for bits and qubits that are much more efficient than any others known, allowing roughly $\log d$ qubits to be hidden in $2 \log d$ qubits. The method can also be used to exhibit the existence of quantum states with locked classical correlations, an arbitrarily large amplification of the correlation being accomplished by sending a negligibly small classical key. Our construction also provides the basic building block for a method of remotely preparing arbitrary $d$-dimensional pure quantum states using approximately $\log d$ bits of communication and $\log d$ ebits of entanglement.

PACS numbers: 03.65.Ta, 03.67.Hk

Keywords: randomization,quantum cryptography,private quantum channel, data hiding,locking correlations, entropic uncertainty relations
\end{abstract}

\section{INTRODUCTION}

In this paper we revisit the question of finding the minimal resources required to randomize a quantum state. This problem has previously been investigated in several variations, always with the conclusion that in order to randomize or, more generally, encrypt a quantum state of $l$ qubits, $2 l$ classical bits of random key are required [1, 2, 3]. This factor of 2 represents a familiar and even welcome phenomenon in quantum information theory; the reason for its appearance is intimately connected to the existence of superdense coding [3, 4]. All this previous work, however, considered only the task of perfectly encrypting quantum states. Here we focus on the task of approximately encrypting quantum states, allowing a negligible but non-zero amount of information to remain available to an eavesdropper. In sharp contrast to the exact case, in this setting we find that the factor of 2 disappears entirely: an $l$-qubit quantum state can be approximately encrypted using $l+o(l)$ bits of random key.

\footnotetext{
*Electronic address: patrick@cs.caltech.edu

${ }^{\dagger}$ Electronic address: wcleung@cs.caltech.edu

${ }^{\ddagger}$ Electronic address: shor@research.att.com

§Electronic address: winter@cs.bris.ac.uk
} 
Our encryption or, more specifically, randomization scheme also exposes a previously unobserved difference between classical and quantum correlations: classical correlations are always effectively destroyed by a local randomization procedure whereas quantum correlations need not be. Therefore, any correlation that survives local randomization must be "nonlocal" and, hence, quantum mechanical in nature. This basic insight provides the intuition behind a new scheme for data hiding in a bipartite system [5, 6, 7]. The encoding is an approximate randomization procedure applied collectively to the two $l$-qubit shares of a $2 l$-qubit system, calibrated to eliminate all correlations that can be detected by local operations and classical communication (LOCC). The failure of the encoding to destroy quantum correlations is striking: there exists a collective decoding operation that recovers all the states on an $[l-o(l)]$-qubit subsystem of the input. In other words, randomization can be used to construct schemes for LOCC hiding of roughly $l$ qubits in a $2 l$-qubit quantum state. This construction is far more efficient than any previously known for hiding qubits or even classical bits.

Another variation on our basic construction can be used to find quantum states whose classical correlations are large but locked. Roughly speaking, this means that local measurements can yield classical data with only a small amount of correlation but that local operations supplemented with a small amount of communication can yield a disproportionately large amount of correlation. The existence of such states was demonstrated in Ref. [8] but some central questions about the range of possible effects were left open. In particular, the authors defined two figures of merit, one measuring the (reciprocal of) amplification of the correlation and the other the ratio of the amount of communication to the amount of unlocked correlation. We give the first demonstration that both quantities can go to zero simultaneously. While seemingly esoteric, the existence of this phenomenon has important implications for the definition of security in quantum cryptographic scenarios; in particular, it establishes the potentially enormous volatility of accessible information. There is also an alternative interpretation of this result: we prove that random observables typically obey extremely strong entropic uncertainty relations.

One final application of the ability to randomize an $l$-qubit state using only $l+o(l)$ random key bits has a sufficiently different character that we present it in a separate paper [9]. Insofar as teleportation [10], or more generally remote state preparation [11], can be interpreted as a method for encrypting quantum states [12, 13], our approximate encryption procedure should give rise to an approximate remote state preparation method consuming only half as much communication as teleportation. We report in [9] how our results on randomization provide the basic building block for a protocol capable of sending an arbitrary pure $l$-qubit quantum state using only $l$ ebits and $l+o(l)$ bits of classical communication.

The rest of the paper is structured as follows. Section Idescribes our results on the private quantum channel, which serve as a prototype for the rest of the paper. Section III then studies approximately randomizing maps by characterizing their effect on classical and quantum correlations. These observations are then put to use in slightly modified form in section IV] which describes our quantum data hiding protocol and proves both its correctness and security. Section $\nabla$ then formalizes the idea of locking classical correlations and describes our contribution. We also include an appendix establishing some results on randomization procedures using subsets of the unitary group, such as the Pauli matrices.

We use the following conventions throughout the paper. log and exp are always taken base 2. Unless otherwise stated, a "state" can be pure or mixed. The symbol for a state (such as $\varphi$ or $\rho$ ) also denotes its density matrix. "Part of an entangled state" refers to a (mixed) state whose purification is accessible to some of the parties. We will make an explicit indication when referring to a pure state. The density operator $|\varphi\rangle\langle\varphi|$ of the pure state $|\varphi\rangle$ will frequently be written simply as $\varphi \cdot \mathcal{B}\left(\mathbb{C}^{d}\right)$ will be used to denote the set of linear operators from $\mathbb{C}^{d}$ to itself and $\mathrm{U}(d) \subset \mathcal{B}\left(\mathbb{C}^{d}\right)$ the unitary group on $\mathbb{C}^{d}$. 
Finally, a word of warning about the cryptographic interpretation of our results. When we say that a scheme for approximate encryption or quantum data hiding is secure for mixed states, it should be assumed that the purifications of those mixed states are inaccessible to all parties considered. Indeed, the possibility that purification-inaccessible security criteria can hold even as purification-accessible criteria fail is essentially a quantum mechanical re-statement of a familiar cryptographic observation: approximate security is sometimes much easier to achieve than perfect security. We exploit the gap throughout this paper.

\section{AN APPROXIMATE PRIVATE QUANTUM CHANNEL}

We consider an insecure one-way quantum channel between two parties Alice and Bob that is noiseless in the absence of eavesdropping. This channel can be made secure against eavesdropping if Alice and Bob are allowed the extra resource of shared random secret key bits. For instance, one can encrypt a state $|\varphi\rangle \in \mathbb{C}^{d}$ using a secret key of length $2 \log d$ as follows [2, 3]. Fix a basis $\{|1\rangle, \ldots,|d\rangle\}$ for $\mathbb{C}^{d}$ and let

$$
X|j\rangle=|(j+1) \bmod d\rangle \text { and } Z|j\rangle=e^{2 \pi i j / d}|j\rangle .
$$

It's straightforward to verify that

$$
\frac{1}{d^{2}} \sum_{j=1}^{d} \sum_{k=1}^{d} X^{j} Z^{k} \varphi Z^{k^{\dagger}} X^{j^{\dagger}}=\frac{\mathbb{I}}{d}
$$

for all states $\varphi$. If $j$ and $k$ are selected using a shared secret key, Alice can encrypt the state using the unitary operation $X^{j} Z^{k}$ and Bob can decrypt by applying $Z^{-k} X^{-j}$. By Eq. (2), the view of an eavesdropper without access to $j$ and $k$ is $\mathbb{I} / d$, which is independent of the input state $\varphi$. This structure, consisting of a set of encoding maps and decoding maps indexed by key values, such that the average encoded state is independent of the input, is known as a private quantum channel for $\mathbb{C}^{d}$. (See Ref. [3] for a formal definition.) In fact, if perfect security is required, it can be shown that the secret key must have length at least $2 \log d[1,2,3,13]$. Here we relax the security criterion.

Definition II.1 A completely positive, trace-preserving (CPTP) map $R: \mathcal{B}\left(\mathbb{C}^{d}\right) \rightarrow \mathcal{B}\left(\mathbb{C}^{d}\right)$ is $\epsilon$ randomizing if, for all states $\varphi$,

$$
\left\|R(\varphi)-\frac{\mathbb{I}}{d}\right\|_{\infty} \leq \frac{\epsilon}{d}
$$

In the above, $\|\cdot\|_{\infty}$ is the operator norm, so Eq. (3) is equivalent to all the eigenvalues of $R(\varphi)$ lying in the interval $[(1-\epsilon) / d,(1+\epsilon) / d]$. By convexity of the norm, it suffices to check the condition for all pure states, a fact we will use repeatedly. In quantum information, distinguishability is frequently measured using the trace norm $\|\cdot\|_{1}=\operatorname{Tr}|\cdot|$, the analogue of variation distance in probability theory. Note that Eq. (3) automatically implies the weaker estimate $\|R(\varphi)-\mathbb{I} / d\|_{1} \leq \epsilon$. The main result of this section is

Theorem II.2 For all $\epsilon>0$ and sufficiently large $d\left(>\frac{10}{\epsilon}\right)$, there exists a choice of unitaries in $U(d)$, $\left\{U_{j}: 1 \leq j \leq n\right\}$ with $n=134 d(\log d) / \epsilon^{2}$ such that the map

$$
R(\varphi)=\frac{1}{n} \sum_{j=1}^{n} U_{j} \varphi U_{j}^{\dagger}
$$

on $\mathcal{B}\left(\mathbb{C}^{d}\right)$ is $\epsilon$-randomizing. 


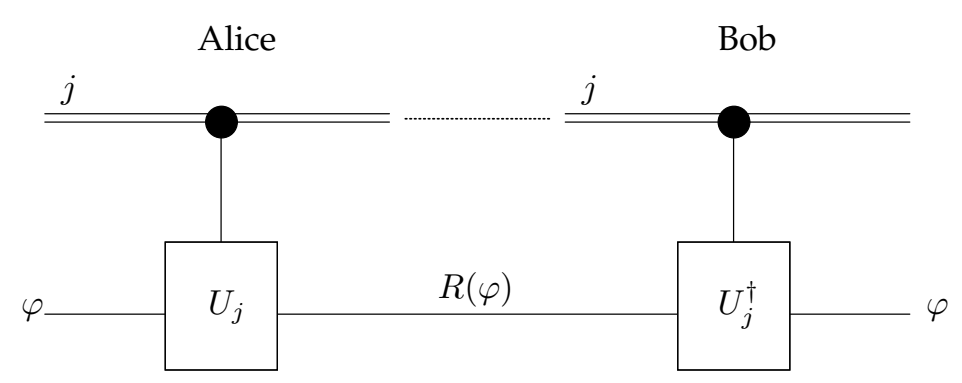

FIG. 1: A private quantum channel built on the randomization map $R$. Alice and Bob share knowledge of the secret key $j$, using it to encrypt and decrypt the state. Because an eavesdropper does not have access to $j$, her view is $R(\varphi) \approx \mathbb{I} / d$. If $\varphi$ is a $d$-dimensional quantum state, then the key length need only be $\log d+o(\log d)$.

As illustrated in figure1 by having Alice and Bob select $j$ using a shared secret key, this map $R$ can be used to build a private quantum channel with key length $\log n=\log d+\log \log d+\log \left(1 / \epsilon^{2}\right)+8$, albeit one that is not perfectly secure, only nearly so. The view of an eavesdropper without access to the key, given a particular input state $\varphi$, is precisely $R(\varphi)$. Definition 1.1 , therefore, doubles as a definition of security. For any distribution of states $\left\{p_{i}, \varphi_{i}\right\}$ supported on $\mathbb{C}^{d}$ we can bound the mutual information accessible to an eavesdropper who performs measurements on the encrypted states $R\left(\varphi_{i}\right)$. This accessible information is bounded above by the Holevo quantity [14]

$$
\begin{aligned}
\chi & =S\left(\sum_{i} p_{i} R\left(\varphi_{i}\right)\right)-\sum_{i} p_{i} S\left(R\left(\varphi_{i}\right)\right) \\
& \leq \log d-\sum_{i} p_{i} S\left(R\left(\varphi_{i}\right)\right) \\
& \leq \log (1+\epsilon) \leq \epsilon /(\ln 2) .
\end{aligned}
$$

The second inequality is true because the definition of $\epsilon$-randomizing maps implies that $R\left(\varphi_{i}\right) \leq$ $(1+\epsilon) \mathbb{I} / d$, which allows for an application of the monotonicity of log. In particular, for all $\alpha>0$, choosing $\epsilon=(\log d)^{-\alpha}$ implies that $\chi \stackrel{d \rightarrow \infty}{\longrightarrow} 0$. In this case, $n=134 d(\log d)^{(1+2 \alpha)}$, so the key size can be taken to be $\log d+(1+2 \alpha) \log \log d+8$. Alternatively, one can choose an arbitrarily small $\alpha>0$, and let $\epsilon=d^{-\alpha}$. Then, $\chi \leq d^{-\alpha} /(\ln 2)$ which is exponentially decaying in the number of qubits to be encrypted, $\log d$. This comes at the cost of a slight increase in the asymptotic key length: $\log n=(1+2 \alpha) \log d+\log \log d+8$. Moreover, as is common with probabilistic existence proofs, it is possible to ensure that the overwhelming majority of random choices succeed at only minor additional cost; in this case, $\log n$ would need to be increased by a constant number of bits for any fixed probability of success. Analogous statements hold for our other constructions later in the paper.

The proof of theorem $\llbracket .2$ is based on a large deviation estimate, lemma $\llbracket .3$, and discretization via a net construction, lemma II.4 both of which will be re-used in other applications later in the paper. The large deviation estimate, in turn, is based on Cramér's theorem (see Ref. [15], for example, for a detailed exposition), which states that for independent, identically distributed 
(i.i.d.) real-valued random variables, $X, X_{1}, X_{2}, \ldots, X_{n}$,

$$
\begin{aligned}
& \operatorname{Pr}\left(\frac{1}{n} \sum_{j=1}^{n} X_{j} \geq a\right) \leq \exp \left(-n \frac{1}{\ln 2} \inf _{x \geq a} \Lambda^{*}(x)\right) \text { and } \\
& \operatorname{Pr}\left(\frac{1}{n} \sum_{j=1}^{n} X_{j} \leq a\right) \leq \exp \left(-n \frac{1}{\ln 2} \inf _{x \leq a} \Lambda^{*}(x)\right)
\end{aligned}
$$

where

$$
\Lambda^{*}(x)=\sup _{\lambda \in \mathbb{R}}\left[\lambda x-\ln \mathbb{E} e^{\lambda X}\right] .
$$

$\Lambda^{*}(x)$ is known as the rate function and $\mathbb{E} e^{\lambda X}$ the moment generating function. (In fact, the harder part of Cramér's theorem deals with the optimality of the rate function. We only need bounds (8) and (9) here, which are surprisingly easy to prove: they require only the Bernstein trick and Markov's inequality.)

Lemma II.3 Let $\varphi$ be a pure state, $P$ a rank p projector and let $\left(U_{j}\right)_{j \geq 1}$ be an i.i.d. sequence of $U(d)$-valued random variables, distributed according to the Haar measure. There exists a constant $C\left(C \geq(6 \ln 2)^{-1}\right)$ such that if $0<\epsilon<1$,

$$
\operatorname{Pr}\left(\left|\frac{1}{n} \sum_{j=1}^{n} \operatorname{Tr}\left(U_{j} \varphi U_{j}^{\dagger} P\right)-\frac{p}{d}\right| \geq \frac{\epsilon p}{d}\right) \leq 2 \exp \left(-C n p \epsilon^{2}\right) .
$$

Proof Since the Haar measure is left and right invariant, we may assume that $\varphi=|1\rangle\langle 1|$ and $P=$ $\sum_{i=1}^{p}|i\rangle\langle i|$ for some fixed orthonormal basis $\{|i\rangle\}$. Let $\left|g_{j}\right\rangle=\sum_{i=1}^{d} g_{i j}|i\rangle$, where the i.i.d. complex random variables $g_{i j} \sim N_{\mathbb{C}}(0,1)$. (That is, the real and imaginary parts of $g_{i j}$ are independent gaussian random variables with mean 0 and variance 1/2.) The distribution of $\left|g_{j}\right\rangle$ is the same as the distribution for $\left\|g_{j}\right\|_{2} U_{j}|1\rangle$. For a fixed $j$, let $U=U_{j}$ and $|g\rangle=\left|g_{j}\right\rangle$. The convexity of exp implies that

$$
\begin{aligned}
\mathbb{E}_{g} \exp \left(\frac{\lambda}{d} \sum_{i=1}^{p}|\langle i \mid g\rangle|^{2}\right) & =\mathbb{E}_{U} \mathbb{E}_{g} \exp \left(\frac{\lambda\|g\|_{2}^{2}}{d} \sum_{i=1}^{p}|\langle i|U| 1\rangle|^{2}\right) \\
& \geq \mathbb{E}_{U} \exp \left(\mathbb{E}_{g} \frac{\lambda\|g\|_{2}^{2}}{d} \sum_{i=1}^{p}|\langle i|U| 1\rangle|^{2}\right) \\
& =\mathbb{E}_{U} \exp \left(\lambda \sum_{i=1}^{p}|\langle i|U| 1\rangle|^{2}\right) \\
& =\mathbb{E}_{U} \exp \left(\lambda \operatorname{Tr}\left(U \varphi U^{\dagger} P\right)\right) .
\end{aligned}
$$

This inequality between moment generating functions establishes, via Cramér's theorem, that the rate function $\Lambda_{U}^{*}$ for the random variable $\operatorname{Tr}\left(U \varphi U^{\dagger} P\right)$ and the rate function $\Lambda_{p}^{*}$ for $\frac{1}{d} \sum_{i=1}^{p}|\langle i \mid g\rangle|^{2}$ are related by the inequality $\Lambda_{p}^{*}(x) \leq \Lambda_{U}^{*}(x)$. It follows from the definitions that $\Lambda_{p}^{*}(p x / d)$, in turn, is equal to $p \Lambda_{g}^{*}(x)$, where $\Lambda_{g}^{*}$ is the rate function for $\left|g_{i j}\right|^{2}$. Therefore,

$$
\operatorname{Pr}\left(\frac{1}{n} \sum_{j=1}^{n} \operatorname{Tr}\left(U_{j} \varphi U_{j}^{\dagger} P\right)-\frac{p}{d} \geq \frac{\epsilon p}{d}\right) \leq \exp \left(-n p \frac{1}{\ln 2} \inf _{x \geq \epsilon} \Lambda_{g}^{*}(1+x)\right) .
$$


The rate function $\Lambda_{g}^{*}$ can be evaluated directly, with the result that $\Lambda_{g}^{*}(1+\epsilon) \geq C \epsilon^{2}$, where $C$ can be chosen to be the constant $(6 \ln 2)^{-1}$ [9]. Repeating the argument for deviations below the mean and applying the union bound completes the proof.

As an aside, we note that the probability density function for $\operatorname{Tr}\left(U_{j} \varphi U_{j}^{\dagger} P\right)$ was recently calculated exactly by Zyczkowski and Sommers [16]. In principle, this should allow for an exact calculation of the rate function $\Lambda_{U}^{*}$.

Lemma II.4 For $0<\epsilon<1$ and $\operatorname{dim} \mathcal{H}=d$ there exists a set $\mathcal{M}$ of pure states in $\mathcal{H}$ with $|\mathcal{M}| \leq(5 / \epsilon)^{2 d}$, such that for every pure state $|\varphi\rangle \in \mathcal{H}$ there exists $|\tilde{\varphi}\rangle \in \mathcal{M}$ with $\||\varphi\rangle\langle\varphi|-| \tilde{\varphi}\rangle\langle\tilde{\varphi}| \|_{1} \leq \epsilon$. (We call such a set an $\epsilon$-net.)

Proof We begin by relating the trace norm to the Hilbert space norm:

$$
\begin{aligned}
\||\tilde{\varphi}\rangle-|\varphi\rangle \|_{2}^{2} & =2-2 \operatorname{Re}\langle\tilde{\varphi} \mid \varphi\rangle \\
& \geq 1-|\langle\tilde{\varphi} \mid \varphi\rangle|^{2} \\
& =\left(\frac{1}{2} \||\tilde{\varphi}\rangle\langle\tilde{\varphi}|-| \varphi\rangle\langle\varphi| \|_{1}\right)^{2},
\end{aligned}
$$

where the last line can be shown by evaluating the eigenvalues of $|\tilde{\varphi}\rangle\langle\tilde{\varphi}|-| \varphi\rangle\langle\varphi|$. Thus it will be sufficent to find an $\epsilon / 2$-net for the Hilbert space norm. Let $\mathcal{M}=\left\{\left|\varphi_{i}\right\rangle: 1 \leq i \leq m\right\}$ be a maximal set of pure states satisfying $\|\left|\varphi_{i}\right\rangle-\left|\varphi_{j}\right\rangle \|_{2} \geq \epsilon / 2$ for all $i$ and $j$. (Such a set exists by Zorn's lemma.) By definition, $\mathcal{M}$ is an $\epsilon / 2$-net for $\|\cdot\|_{2}$. We can then estimate $m$ by a volume argument. As subsets of $\mathbb{R}^{2 d}$, the open balls of radius $\epsilon / 4$ about each $\left|\varphi_{i}\right\rangle$ are pairwise disjoint and all contained in the ball of radius $1+\epsilon / 4$ centered at the origin. Therefore,

$$
m(\epsilon / 4)^{2 d} \leq(1+\epsilon / 4)^{2 d} .
$$

Proof (Of theorem $\amalg .2$ Let $\left(U_{j}\right)_{j \geq 1}$ be i.i.d. U(d)-valued random variables, distributed according to the Haar measure. We will show that with high probability the corresponding $R$ in Eq. (4) is $\epsilon$-randomizing. The proof will consist of bounding

$$
\operatorname{Pr}_{U}\left(\sup _{\varphi}\left\|\frac{1}{n} \sum_{j=1}^{n} U_{j} \varphi U_{j}^{\dagger}-\frac{\mathbb{I}}{d}\right\|_{\infty} \geq \frac{\epsilon}{d}\right)=\underset{U}{\operatorname{Pr}}\left(\sup _{\varphi} \sup _{\psi}\left|\frac{1}{n} \sum_{j=1}^{n} \operatorname{Tr}\left(U_{j} \varphi U_{j}^{\dagger} \psi\right)-\frac{1}{d}\right| \geq \frac{\epsilon}{d}\right) .
$$

The optimizations over $\varphi$ and $\psi$ can both be taken over pure states only by the convexity of $|\cdot|$. Fix a net of projectors $\mathcal{M}=\{X\}$ and let $\tilde{\varphi}$ be the net point corresponding to $\varphi$ so that

$$
\sup _{\varphi}\|\varphi-\tilde{\varphi}\|_{1} \leq \frac{\epsilon}{2 d}
$$


Define $\tilde{\psi}$ similarly. Lemma $\llbracket$ provides a net with $|\mathcal{M}| \leq\left(\frac{10 d}{\epsilon}\right)^{2 d}$. We can then proceed as follows:

$$
\begin{array}{r}
\operatorname{Pr}_{U}\left(\sup _{\varphi} \sup _{\psi}\left|\frac{1}{n} \sum_{j=1}^{n} \operatorname{Tr}\left(U_{j} \varphi U_{j}^{\dagger} \psi\right)-\frac{1}{d}\right| \geq \frac{\epsilon}{d}\right) \\
\leq \underset{U}{\operatorname{Pr}}\left(\sup _{\varphi} \sup _{\psi} \frac{1}{n} \sum_{j=1}^{n}\left|\operatorname{Tr}\left(U_{j} \varphi U_{j}^{\dagger} \psi\right)-\operatorname{Tr}\left(U_{j} \tilde{\varphi} U_{j}^{\dagger} \tilde{\psi}\right)\right|\right. \\
\left.+\left|\frac{1}{n} \sum_{j=1}^{n} \operatorname{Tr}\left(U_{j} \tilde{\varphi} U_{j}^{\dagger} \tilde{\psi}\right)-\frac{1}{d}\right| \geq \frac{\epsilon}{d}\right) \\
\leq \operatorname{Pr}_{U}\left(\sup _{\varphi} \sup _{\psi}\left|\frac{1}{n} \sum_{j=1}^{n} \operatorname{Tr}\left(U_{j} \tilde{\varphi} U_{j}^{\dagger} \tilde{\psi}\right)-\frac{1}{d}\right| \geq \frac{\epsilon}{2 d}\right) .
\end{array}
$$

In the last inequality, we used the estimate

$$
\left|\operatorname{Tr}\left(U_{j} \varphi U_{j}^{\dagger} \psi\right)-\operatorname{Tr}\left(U_{j} \tilde{\varphi} U_{j}^{\dagger} \tilde{\psi}\right)\right| \leq\|\varphi-\tilde{\varphi}\|_{\infty}+\|\psi-\tilde{\psi}\|_{\infty},
$$

which, because $\varphi-\tilde{\varphi}$ and $\psi-\tilde{\psi}$ are traceless and either zero or rank 2 , is equal to $\frac{1}{2}\|\varphi-\tilde{\varphi}\|_{1}+$ $\frac{1}{2}\|\psi-\tilde{\psi}\|_{1}$. This sum is then less than or equal to $\epsilon /(2 d)$ by construction of the net.

Next, we replace the optimization over the set of all pure states in Eq. (22) by optimization over the net, use the union bound and apply lemma

$$
\begin{aligned}
& \operatorname{Pr}_{U}\left(\max _{\tilde{\varphi}, \tilde{\psi} \in \mathcal{M}}\left|\frac{1}{n} \sum_{j=1}^{n} \operatorname{Tr}\left(U_{j} \tilde{\varphi} U_{j}^{\dagger} \tilde{\psi}\right)-\frac{1}{d}\right| \geq \frac{\epsilon}{2 d}\right) \\
\leq & |\mathcal{M}|^{2} \max _{\tilde{\varphi}, \tilde{\psi} \in \mathcal{M}} \operatorname{Pr}_{U}\left(\left|\frac{1}{n} \sum_{j=1}^{n} \operatorname{Tr}\left(U_{j} \tilde{\varphi} U_{j}^{\dagger} \tilde{\psi}\right)-\frac{1}{d}\right| \geq \frac{\epsilon}{2 d}\right) \\
\leq & \left(\frac{10 d}{\epsilon}\right)^{4 d} \exp \left(-\frac{C n \epsilon^{2}}{4}\right),
\end{aligned}
$$

where $C \geq(6 \ln 2)^{-1}$ is the same constant as in the proof of lemma $\llbracket .3$ The existence of the desired $\epsilon$-randomizing map is guaranteed if the above probability is bounded away from 1, which is the case if $n>\frac{16 d}{C \epsilon^{2}} \log \left(\frac{10 d}{\epsilon}\right)$. If $d>\frac{10}{\epsilon}$, this is true when when $n \geq 192(\ln 2) \epsilon^{-2} d \log d$.

\section{RANDOMIZATION AND THE DESTRUCTION OF CORRELATIONS}

Our discussion in the previous section demonstrates that a quantum operation $R$ constructed by averaging over $134 d(\log d) / \epsilon^{2}$ randomly selected unitaries will be $\epsilon$-randomizing with high probability. In this section, our goal will be to investigate the properties of general $\epsilon$-randomizing maps so the method used to construct $R$ will be immaterial as long as

$$
\left\|R(\varphi)-\frac{\mathbb{I}}{d}\right\|_{\infty} \leq \frac{\epsilon}{d}
$$

for all $\varphi$. The definition, it should be noticed, makes no mention of the effect of $R$ on a system that is correlated with another system (we call this the "environment"). Here we will analyze that effect, which will ultimately lead to a partial characterization of all $\epsilon$-randomizing maps. 
To start, it is easy to verify that an $\epsilon$-randomizing $R$ properly destroys classical correlations between the system being randomized and its environment:

Lemma III.1 Let $\rho^{A B}=\sum_{i} p_{i} \varphi_{i}^{A} \otimes \psi_{i}^{B}$ be a separable state and $R$ an $\epsilon$-randomizing map on $A$. Then

$$
\left\|(R \otimes I)\left(\rho^{A B}\right)-\frac{\mathbb{I}}{d} \otimes \rho^{B}\right\|_{1} \leq \epsilon .
$$

Proof This is straightforward:

$$
\begin{aligned}
\left\|(R \otimes I)\left(\rho^{A B}\right)-\frac{\mathbb{I}}{d} \otimes \rho^{B}\right\|_{1} & =\left\|\sum_{i} p_{i}\left[R\left(\varphi_{i}^{A}\right) \otimes \psi_{i}^{B}-\frac{\mathbb{I}}{d} \otimes \psi_{i}^{B}\right]\right\|_{1} \\
& \leq \sum_{i} p_{i}\left\|R\left(\varphi_{i}^{A}\right) \otimes \psi_{i}^{B}-\frac{\mathbb{I}}{d} \otimes \psi_{i}^{B}\right\|_{1} \\
& =\sum_{i} p_{i}\left\|R\left(\varphi_{i}^{A}\right)-\frac{\mathbb{I}}{d}\right\|_{1} \\
& \leq \epsilon .
\end{aligned}
$$

Thus, for classically correlated states, approximate randomization implies the destruction of correlations with other systems. Indeed, for classically correlated states, finding an operation that will destroy correlations is effectively the same thing as finding an operation that will erase local information.

This equivalence fails dramatically for entangled states. (In contrast, any perfectly randomizing map does destroy all possible correlations including entanglement.) Indeed, if we apply an $R$ constructed using the method of theorem $\amalg .2$ to half of a maximally entangled state $|\Phi\rangle=\frac{1}{\sqrt{d}} \sum_{j=1}^{d}|i\rangle|i\rangle$, then the resulting state has rank at most $n=o\left(d^{2}\right)$, so

$$
\left\|(R \otimes I)(\Phi)-\frac{1}{d^{2}} \mathbb{I} \otimes \mathbb{I}\right\|_{1} \leq 2\left(1-n / d^{2}\right) \stackrel{d \rightarrow \infty}{\longrightarrow} 2,
$$

meaning that $(R \otimes I)(\Phi)$ and $\frac{1}{d^{2}} \mathbb{I} \otimes \mathbb{I}$ can be distinguished by a collective measurement with negligible probability of error for large $d$. It goes without saying then that the approximate randomization does not eliminate all the correlations that were originally present in the maximally entangled state. Nonetheless, the correlations that remain have been rendered invisible to local operations and classical communication, recovering at least some of the spirit of lemma

Lemma III.2 Let $R$ be an $\epsilon$-randomizing quantum operation, $M=\left\{M_{i}\right\}$ be a positive operator-valued measure (POVM) that can be implemented using LOCC, $p_{i}:=\operatorname{Tr}\left(M_{i}(R \otimes I)(\Phi)\right)$ and $q_{i}:=\operatorname{Tr}\left(M_{i} \frac{1}{d^{2}} \mathbb{I}\right)$. Then $\|p-q\|_{1} \leq \epsilon$.

Proof If $M$ can be implemented using LOCC then it will have the separable form $M_{i}=X_{i} \otimes Y_{i}$. Moreover, we can assume without loss of generality that $X_{i}$ and $Y_{i}$ are rank 1 since refining the measurement can only increase the $\ell_{1}$ distance between the outcome probability distributions. Making use of the identities $Y_{i} \operatorname{Tr} Y_{i}=Y_{i}^{2}$ and $\left(I \otimes Y_{i}\right) \Phi\left(I \otimes Y_{i}\right)=\frac{1}{d} Y_{i}^{T} \otimes Y_{i}$, the demonstration is 
then direct:

$$
\begin{aligned}
\|p-q\|_{1} & =\sum_{i}\left|\operatorname{Tr}\left[\left(X_{i} \otimes Y_{i}\right)(R \otimes I)(\Phi)\right]-\operatorname{Tr}\left[\left(X_{i} \otimes Y_{i}\right) \frac{\mathbb{I}}{d^{2}}\right]\right| \\
& =\sum_{i} \mid \operatorname{Tr}\left[\left(X_{i} \otimes \mathbb{I}\right)(R \otimes I)\left(\frac{Y_{i}^{T}}{\operatorname{Tr} Y_{i}^{T}} \otimes \frac{Y_{i}}{d}\right)-\operatorname{Tr}\left[\left(X_{i} \otimes Y_{i}\right) \frac{\mathbb{I}}{d^{2}}\right] \mid\right. \\
& =\sum_{i}\left|\operatorname{Tr}\left[X_{i}\left(R\left(\frac{Y_{i}^{T}}{\operatorname{Tr} Y_{i}^{T}}\right)-\frac{\mathbb{I}}{d}\right)\right]\right|\left|\operatorname{Tr} \frac{Y_{i}}{d}\right| \\
& \leq \sum_{i} \frac{\epsilon}{d^{2}} \operatorname{Tr}\left(X_{i}\right) \operatorname{Tr}\left(Y_{i}\right) \leq \epsilon,
\end{aligned}
$$

where in the last step we have used that $\sum_{i} X_{i} \otimes Y_{i}=\mathbb{I}$ and the fact that $R$ is $\epsilon$-randomizing.

A very similar proof demonstrates the more general

Theorem III.3 Let $M=\left\{M_{i}\right\}$ be a POVM that can be implemented using LOCC, $p_{i}:=\operatorname{Tr}\left(M_{i}(R \otimes\right.$ $\left.I)\left(\rho^{A B}\right)\right)$ and $q_{i}:=\operatorname{Tr}\left(M_{i}\left(\frac{1}{d} \mathbb{I} \otimes \rho^{B}\right)\right)$. Then $\|p-q\|_{1} \leq \epsilon$.

Thus, while some kind of correlation can persevere when half of an entangled state is randomized, that correlation will all be inaccessible to LOCC measurements. In fact, the proof shows that it will be inaccessible to all measurements that can be implemented using separable superoperators. It's tempting to speculate that lemma $\amalg$.2 provides a characterization of all $\epsilon$-randomizing operations. There is at least a weak sense in which that is true: if the conclusion of lemma III.2 holds for a map $R$, then for all states $\varphi$,

$$
\left\|R(\varphi)-\frac{\mathbb{I}}{d}\right\|_{1} \leq \epsilon
$$

Recall that this condition is weaker than the operator norm definition of $\epsilon$-randomization that we use, however. This might suggest that our definition is too strong and that this trace norm version might be more easily characterized. Unfortunately, our proof of lemma $\amalg$ makes explicit use of the stronger condition. We don't know if it would hold for $R$ only satisfying Eq. (38). The fact that $\epsilon$-randomizing maps render the correlations of entanglement invisible to LOCC also raises the question of their relationship to the phenomenon of quantum nonlocality without entanglement [17]. The range of connections between $\epsilon$-randomizing maps and the physics of nonlocality will be further developed in a upcoming paper [18].

\section{QUANTUM DATA HIDING}

Theorem ЩII.3 also immediately suggests another application of $\epsilon$-randomizing maps: quantum data hiding, the name given to schemes for sharing bits or qubits between multiple parties in such a way that the data cannot be accessed by LOCC operations alone. We will focus on the bipartite case, where our methods provide a protocol for hiding $l$ qubits in a bipartite state of roughly $2 l$ qubits. This is far more efficient than previous constructions for hiding either bits or qubits, where the best previous constructions gave ratios that depended on the security requirements. To achieve $\delta=\epsilon=1 / 16$ in terms of the parameters introduced below, for example, the separable Werner state construction of Ref. [5] requires roughly 24 qubits per hidden bit. (Using, in the notation of that paper, $K=4$ and $d=64$.) To achieve qubit hiding, the construction of Ref. [6] would have multiplied that overhead by a further dimension-dependent factor. (We note that by making additional assumptions about the operations available to the parties, it is possible to improve on the $1: 2$ ratio between hidden and physical qubits. It was recently discovered, for example, that in some systems with superselection rules, a ratio of $1: 1$ is achievable [19].) 
Definition IV.1 (Adapted from Ref. [6]) $A(\delta, \epsilon, p, q)$-qubit hiding scheme consists of a CPTP encoding map $E: \mathcal{B}\left(\mathbb{C}^{p}\right) \rightarrow \mathcal{B}\left(\mathbb{C}^{q}\right)$ and a $C P T P$ decoding map $D: \mathcal{B}\left(\mathbb{C}^{q}\right) \rightarrow \mathcal{B}\left(\mathbb{C}^{p}\right)$ such that

1. (Security) For all LOCC measurements $L$, as well as all states $\varphi_{0}$ and $\varphi_{1}$ on $\mathbb{C}^{p}$,

$$
\left\|L\left(E\left(\varphi_{0}\right)\right)-L\left(E\left(\varphi_{1}\right)\right)\right\|_{1} \leq \epsilon
$$

2. (Correctness) For all states $\varphi$ on $\mathbb{C}^{p},\|(D \circ E)(\varphi)-\varphi\|_{1} \leq \delta$.

The security criterion obviously implicitly assumes some bipartite structure $\mathbb{C}^{q} \cong \mathcal{H}_{A} \otimes \mathcal{H}_{B}$. In our constructions, we will set $\operatorname{dim}\left(\mathcal{H}_{A}\right)=\operatorname{dim}\left(\mathcal{H}_{B}\right)=d$. Our main result is

Theorem IV.2 For all $\delta, \epsilon>0$ (satisfying $\left.\epsilon^{2} \log \left(40 / \delta^{2}\right)<1\right)$ and sufficiently large $d(d>$ $\left.\max \left\{\frac{36}{C \delta^{2}}, \sqrt{15 / \epsilon}, 21\right\}\right)$, one can construct a $\left(\delta, \epsilon, p, d^{2}\right)$-qubit hiding scheme, with

$$
p=\frac{C \delta^{2} \epsilon^{2} d}{1188 \log d} .
$$

and $C=(6 \ln 2)^{-1}$. The encoding map is given by

$$
R(\rho)=\frac{1}{n} \sum_{j=1}^{n} U_{j} \rho U_{j}^{\dagger}
$$

where $\left\{U_{j}\right\} \subset U\left(d^{2}\right)$, and $n=\frac{99}{C \epsilon^{2}} d \log d$.

The main point, unfortunately obscured by the proliferation of constants and conditions is simply this: for these hiding schemes, the limiting ratio of hidden qubits to physical qubits is

$$
\lim _{d \rightarrow \infty} \frac{\log p}{\log d^{2}}=\frac{1}{2}
$$

The idea behind our approach is simple. We randomly choose the $U_{j}$ acting on the $A B$ system. With $n$ sufficiently large, we can assure that for all $\rho, R(\rho)$ is effectively indistinguishable (by LOCC alone) from the maximally mixed state on $A B$. Then we restrict the input $\rho$ to have support on a sufficiently small subspace $S$ to ensure that the subspaces $\left\{U_{j} S\right\}$ can be reliably distinguished by a collective measurement on $A B$. This strategy is summarized in figure 2

We will prove the security and correctness in the next two subsections.

\section{A. Security of the protocol}

The proof of security uses techniques similar to those in the proofs of lemmas 【.3 and 【II.2 so we only give the outline here. As usual, let $\left\{U_{j}: 1 \leq j \leq n\right\}$ be $\mathrm{U}\left(d^{2}\right)$-valued independent random variables distributed according to the Haar measure. For $0<\epsilon<1$,

$$
\underset{U}{\operatorname{Pr}}\left(\sup _{\varphi \in S X \otimes Y}\left|\operatorname{Tr}\left[\frac{1}{n} \sum_{j=1}^{n}(X \otimes Y) U_{j} \varphi U_{j}^{\dagger}\right]-\frac{1}{d^{2}}\right| \geq \frac{\epsilon}{d^{2}}\right) \leq 2\left|\mathcal{M}_{d}\right|^{2}\left|\mathcal{M}_{p}\right| \exp \left(-\frac{C n \epsilon^{2}}{4}\right),
$$

where $X$ and $Y$ are $d$-dimensional rank 1 projectors acting on $A$ and $B$ respectively, and $\varphi \in S$ is a $p$-dimensional pure state (to be hidden). $C$ is the same positive constant as in lemma II.3, $\mathcal{M}_{d}$ and $\mathcal{M}_{p}$ are $\frac{\epsilon}{3 d^{2}}$-nets for $d$-dimensional and $p$-dimensional rank 1 projectors respectively. Eq. (43) 
(a)

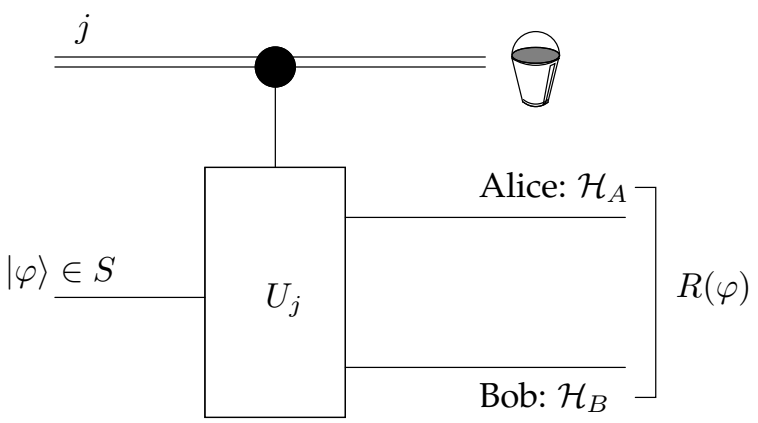

(b)

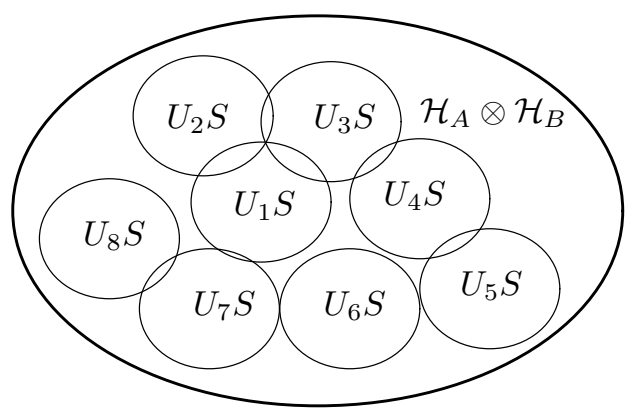

FIG. 2: Quantum data hiding. (a) depicts the encoding procedure. A random $U_{j}$ is applied to the state $|\varphi\rangle$ drawn from subspace $S$. The output, $R(\varphi)$, is almost indistinguishable from the maximally mixed state using LOCC alone. (b) The different subspaces $\left\{U_{j} S\right\}$ have very small overlaps, however, so a collective operation on $\mathcal{H}_{A} \otimes \mathcal{H}_{B}$ can be used to distinguish them without causing much distortion to the encoded states $U_{j}|\varphi\rangle$.

can be proved in a way very similar to lemma I.3. From lemma $\llbracket$ we can choose $\left|\mathcal{M}_{d}\right|^{2}\left|\mathcal{M}_{p}\right|=$ $\left(15 d^{2} / \epsilon\right)^{2(2 d+p)}$. Whenever $d^{2}>\max (15 / \epsilon, 16)$ and $n \geq 33(2 d+p)(\log d) /\left(C \epsilon^{2}\right)$, the probability in Eq. (43) is strictly less than $1 / 2$, in which case more than half of the choices for $\left\{U_{j}\right\}$ are such that for all $\varphi, M$ and $N$,

$$
\left|\operatorname{Tr}\left[\frac{1}{n} \sum_{j=1}^{n}(M \otimes N) U_{j} \varphi U_{j}^{\dagger}\right]-\frac{1}{d^{2}}\right| \leq \frac{\epsilon}{d^{2}} .
$$

To finish the proof of security, we use arguments similar to those in lemma III.2 Let $U_{j}$ be chosen so that $R(\varphi)=\frac{1}{n} \sum_{j=1}^{n} U_{j} \varphi U_{j}^{\dagger}$ satisfies Eq. (44). Let $\left\{X_{i} \otimes Y_{i}\right\}$ be any POVM implemented by LOCC, where $X_{i}$ and $Y_{i}$ are both rank 1 . For any state $\varphi \in S$, let $p_{i}=\operatorname{Tr}\left(\left(X_{i} \otimes Y_{i}\right) R(\varphi)\right)$ and $q_{i}=\operatorname{Tr}\left(\left(X_{i} \otimes Y_{i}\right) \frac{\mathbb{I}}{d^{2}}\right)$. Using Eq. (44) we find

$$
\begin{aligned}
\|p-q\|_{1} & =\sum_{i}\left|\operatorname{Tr}\left(\left(X_{i} \otimes Y_{i}\right) R(\varphi)\right)-\frac{1}{d^{2}} \operatorname{Tr}\left(X_{i} \otimes Y_{i}\right)\right| \\
& \leq \sum_{i} \frac{\epsilon}{d^{2}} \operatorname{Tr}\left(X_{i} \otimes Y_{i}\right) \leq \epsilon .
\end{aligned}
$$

Therefore, when the conditions stated above on $n, d$ and $\epsilon$ are satisfied, the security condition is fulfilled with probability at least $1 / 2$ for a random selection of unitaries.

\section{B. Correctness of the protocol}

To complete the construction of the data hiding scheme, we must also show that the decoding can be performed reliably. Let $S$ be a fixed subspace of dimension $p$ in $\mathcal{H}_{A} \otimes \mathcal{H}_{B}$ and let $P$ be the projector onto $S$. Our decoding procedure will be given by the transpose channel [20], a generalization of the pretty good measurement [21]. Specifically, let $N=\sum_{i=1}^{n} U_{i} P U_{i}^{\dagger}$ and $D_{i}=P U_{i}^{\dagger} N^{-1 / 2}$. Our decoding procedure $D$ will be given by performing the quantum operation with Kraus elements $D_{i}$. Our proof that this decoding procedure works is via a reduction to the task of decoding classical data, for which there are well-known criteria for the success of the pretty good measurement [22]. 
Fixing $0<\alpha<1$, our goal will be to ensure that $\left|\left\langle\varphi\left|D_{i} U_{i}\right| \varphi\right\rangle\right|^{2} \geq 1-\alpha$ for all $i=1, \ldots, n$ and whenever $|\varphi\rangle \in S$. Then, $\langle\varphi|D \circ R(\varphi)| \varphi\rangle \geq 1-\alpha$. It would then follow by standard inequalities [23] that $\|D \circ R(\varphi)-\varphi\|_{1} \leq 2 \sqrt{\alpha}$ and, choosing $\alpha=\delta^{2} / 4$, that the correctness criterion is satisfied. To begin, fix any basis $\mathcal{E}=\{|j\rangle\}$ for $S$. Our strategy is to first show that, for any given $i$ and $|j\rangle$, the operation $D$ decodes $U_{i}|j\rangle$ correctly with high probability by relating $D$ to the pretty good measurement for decoding classical messages. We then show that $D$ succeeds on all pure input states by verifying that it succeeds simultaneously on a large enough set of bases to effectively cover the set of all states.

So, consider decoding the classical messages $i=1, \cdots, n$ and $j=1, \cdots, p$ by applying the pretty good measurement to the set of states $\left\{U_{i}|j\rangle\right\}_{i j}$. The POVM elements are $M_{i j}=$ $N^{-1 / 2} U_{i}|j\rangle\langle j| U_{i}^{\dagger} N^{-1 / 2}$. This POVM can be implemented in two stages: first $D$ is applied and the outcome $i$ is recorded, then the projective measurement onto the basis $\mathcal{E}$ is performed. To see this, observe that

$$
\begin{aligned}
\left\langle j\left|D_{i} \rho D_{i}^{\dagger}\right| j\right\rangle & =\left\langle j\left|P U_{i}^{\dagger} N^{-1 / 2} \rho N^{-1 / 2} U_{i} P\right| j\right\rangle \\
& =\left\langle j\left|U_{i}^{\dagger} N^{-1 / 2} \rho N^{-1 / 2} U_{i}\right| j\right\rangle \\
& =\operatorname{Tr}\left(\rho M_{i j}\right) .
\end{aligned}
$$

In particular, this calculation also demonstrates that

$$
\left|\left\langle j\left|D_{i} U_{i}\right| j\right\rangle\right|^{2}=\operatorname{Tr}\left(U_{i}|j\rangle\langle j| U_{i}^{\dagger} M_{i j}\right)
$$

so the decoding procedure $D$ succeeds on $|j\rangle$ provided that for each $i$, the pretty good measurement identifies $U_{i}|j\rangle$ with high probability. Applying the criterion of Hausladen et al. for the success of the pretty good measurement [22], we find that

$$
1-\left|\left\langle j\left|D_{i} U_{i}\right| j\right\rangle\right|^{2} \leq \Delta_{i j}:=\sum_{i^{\prime} j^{\prime} \neq i j}\left|\left\langle j\left|U_{i}^{\dagger} U_{i^{\prime}}\right| j^{\prime}\right\rangle\right|^{2}
$$

Notice that terms for which $j^{\prime} \neq j$ and $i^{\prime}=i$ do not contribute to $\Delta_{i j}$ so its expectation value is

$$
\begin{aligned}
\mathbb{E}_{U} \Delta_{i j} & =\sum_{i^{\prime} \neq i} \sum_{j^{\prime}} \operatorname{Tr}\left[\mathbb{E}_{U} U_{i}|j\rangle\left\langle j\left|U_{i}^{\dagger} U_{i^{\prime}}\right| j^{\prime}\right\rangle\left\langle j^{\prime}\right| U_{i^{\prime}}^{\dagger}\right] \\
& =\sum_{i^{\prime} \neq i} \sum_{j^{\prime}} \operatorname{Tr}\left[\frac{\mathbb{I}}{d^{2}} \frac{\mathbb{I}}{d^{2}}\right]=\frac{(n-1) p}{d^{2}}
\end{aligned}
$$

which is small provided $n p \ll d^{2}$. We will be interested in

$$
\underset{U}{\operatorname{Pr}}\left(\Delta_{i j} \geq(1+\eta) \frac{(n-1) p}{d^{2}}\right),
$$

which by the left invariance of the Haar measure is equal to

$$
\underset{U}{\operatorname{Pr}}\left(\sum_{i^{\prime} \neq i} \sum_{j^{\prime}}\left|\left\langle j\left|U_{i^{\prime}}\right| j^{\prime}\right\rangle\right|^{2} \geq(1+\eta) \frac{(n-1) p}{d^{2}}\right) .
$$

Invoking lemma $\llbracket .3$ with $\eta=1 / 2$, the state $|j\rangle\langle j|$ and projector $\sum_{j^{\prime}=1}^{p}\left|j^{\prime}\right\rangle\left\langle j^{\prime}\right|$, the probability that $\Delta_{i j}$ exceeds $\frac{3(n-1) p}{2 d^{2}}$ is less than or equal to $\exp (-C(n-1) p / 4)$ for the same positive constant $C$ in 
lemma II.3. By the union bound, the probability that this bad event happens for at least one of the choices of $i$ is less than or equal to $n \exp (-C(n-1) p / 4)$ :

$$
\underset{U}{\operatorname{Pr}}\left(\min _{i}\left|\left\langle j\left|D_{i} U_{i}\right| j\right\rangle\right|^{2} \leq 1-\frac{3(n-1) p}{2 d^{2}}\right) \leq n \exp (-C(n-1) p / 4) .
$$

Now, as discussed earlier, our goal is to verify that $\left|\left\langle\varphi\left|D_{i} U_{i}\right| \varphi\right\rangle\right|^{2}$ will be large for all $|\varphi\rangle \in S$. Fix an $\frac{\alpha}{2}$-net for pure states on $S$. The size of this net can be taken to be less than or equal to $\left(\frac{10}{\alpha}\right)^{2 p}$. Extend each net point $\tilde{\varphi}$ to a basis of $S$. We have

$$
\operatorname{Pr}_{U}\left(\min _{\tilde{\varphi}} \min _{i}\left|\left\langle\tilde{\varphi}\left|D_{i} U_{i}\right| \tilde{\varphi}\right\rangle\right|^{2} \leq 1-\frac{3(n-1) p}{2 d^{2}}\right) \leq\left(\frac{10}{\alpha}\right)^{2 p} n \exp \left(-\frac{C}{4}(n-1) p\right)
$$

by Eq. (56) and the union bound. The probability of $D$ failing on at least one net point is less than $1 / 2$ if $n>\frac{8}{C} \log \left(\frac{10}{\alpha}\right)+\frac{4}{C} \frac{\log 2 n}{p}+1$. Otherwise,

$$
\begin{aligned}
\left|\left\langle\varphi\left|D_{i} U_{i}\right| \varphi\right\rangle\right|^{2} & \geq\left|\left\langle\tilde{\varphi}\left|D_{i} U_{i}\right| \tilde{\varphi}\right\rangle\right|^{2}-\left.||\left\langle\varphi\left|D_{i} U_{i}\right| \varphi\right\rangle\right|^{2}-\left|\left\langle\tilde{\varphi}\left|D_{i} U_{i}\right| \tilde{\varphi}\right\rangle\right|^{2} \mid \\
& \geq 1-\frac{3(n-1) p}{2 d^{2}}-\frac{\alpha}{2}=1-\alpha
\end{aligned}
$$

for all $|\varphi\rangle \in S$ by choosing $3(n-1) p / d^{2}=\alpha$.

That is essentially the end of the proof. All that remains is to make appropriate choices for our various parameters. Collecting all our requirements, we find that the correctness condition $\|D \circ R(\varphi)-\varphi\|_{1} \leq \delta$ for all $\varphi$ is satisfied with probability at least $1 / 2$ if

$$
n>\frac{8}{C} \log \left(\frac{10}{\alpha}\right)+\frac{4}{C} \frac{\log 2 n}{p}+1,
$$

$\alpha=\delta^{2} / 4$ and $3(n-1) p / d^{2}=\alpha$. Recall that the security criterion is satisfied with probability at least $1 / 2$ provided $n \geq 33(2 d+p)(\log d) /\left(C \epsilon^{2}\right)$ and $d>\max (\sqrt{15 / \epsilon}, 4)$. Restricting to $p \leq d$, we make the choice

$$
n=\frac{99}{C \epsilon^{2}} d \log d, \text { and then } p=\frac{C \epsilon^{2} \delta^{2}}{1188} \frac{d}{\log d} .
$$

If, in addition, $d>\max \left(\frac{36}{C \delta^{2}}, 21\right)$ and $\log \left(40 / \delta^{2}\right)<1 / \epsilon^{2}$, a straightforward calculation shows that all our requirements are met. Therefore, by the union bound, the probability that both the correctness and security criteria are satisfied is greater than 0 for a random choice of $R$. As an example, when $\epsilon=\delta=\frac{1}{16}$, and $d$ is sufficiently large, $\log p \geq \log d-\log \log d-30$. Finally, both $\delta$ and $\epsilon$ can be chosen to be any polynomial in $\frac{1}{\log d}$ without affecting the asymptotic efficiency, and can be chosen to be $d^{-\alpha}$ for small $\alpha>0$ in order to achieve security that is exponential in $2 \log d$, the number of physical qubits, at the expense of a slightly reduced asymptotic efficiency $(1-4 \alpha) / 2$.

\section{LOCKING CLASSICAL CORRELATIONS}

Define the maximum classical mutual information that can be obtained by local measurements $X \otimes Y$ on a bipartite state $\rho_{A B}$ as

$$
I_{c}(\rho)=\max _{X \otimes Y} I(x: y),
$$

where $x$ and $y$ are random variables representing the outcomes of measurements $X$ and $Y$ on $\rho_{A B}$ and $I(x: y)$ is equal to $H(x)+H(y)-H(x, y)$ for the Shannon entropy $H$. Now suppose 
that $\rho_{A B}^{\prime}$ is obtained from $\rho_{A B}$ by communicating $l$ classical bits present in Alice's system to Bob. There are natural cryptographic reasons to worry about the relationship between $I_{c}(\rho), I_{c}\left(\rho^{\prime}\right)$ and $l$. Suppose, for example, that an eavesdropper, initially uncorrelated with a quantum state, can extract $I_{c}(\rho)$ bits of mutual information about some secret classical data by performing a measurement on the state. If instead the eavesdropper started with $l$ classical bits potentially correlated with the secret, one would hope that the most the eavesdropper could learn upon performing her measurement would be less than $I_{c}(\rho)+l D$ bits for some constant $D$. The existence of locked classical correlations in the form presented here demonstrates that such bounds fail drastically in general. As a consequence then, it is generally much more prudent to use the Holevo $\chi$ quantity instead of the accessible information when bounding an eavesdropper's information. ( $\chi$ does obey simple bounds of the desired type.)

In their paper introducing the idea of locked classical correlations, DiVincenzo et al. [8] defined two figures of merit,

$$
r_{1}=\frac{I_{c}(\rho)}{I_{c}\left(\rho^{\prime}\right)} \quad \text { and } \quad r_{2}=\frac{l}{I_{c}\left(\rho^{\prime}\right)-I_{c}(\rho)}
$$

Ideally, the two should be small simultaneously: the first is the ratio of the initial to the final information while the second measures the ratio of the "key length" to the unlocked information. In their paper, they found examples for which $\left(r_{1}, r_{2}\right) \sim\left(\frac{1}{2}, \frac{1}{\log d}\right)$ and $\left(r_{1}, r_{2}\right) \sim\left(\frac{1}{2 \log d}, \frac{1}{2}\right)$. Here we show that $r_{1}$ and $r_{2}$ can be made arbitrarily small at the same time, meaning that the amount of information unlocked is large relative both to the amount of information originally available and relative to the number of classical bits communicated from Alice to Bob.

Theorem V.1 For all $\epsilon, \delta>0$ there exist bipartite states $\rho_{A B}$ with $r_{1} \leq \epsilon$ and $r_{2} \leq \delta$. Alice's system may be taken to be a classical system of $\log d+3 \log \log d$ bits and Bob's a quantum system of $\log d$ qubits provided $\epsilon$ is smaller than some fixed constant, $\log d>\frac{16}{C^{\prime \prime} \epsilon} \log \frac{20}{\epsilon}$ (where $C^{\prime \prime}$ is a positive constant) and

$$
\delta \geq \frac{3 \log \log d}{(1-\epsilon / 2) \log d} .
$$

As in the original work, the states we consider will have the form

$$
\rho_{A B}=\frac{1}{d n} \sum_{i=1}^{d} \sum_{j=1}^{n}|i j\rangle\left\langle\left. i j\right|_{A} \otimes\left(U_{j}|i\rangle\langle i| U_{j}^{\dagger}\right)_{B},\right.
$$

where the $\left\{|i j\rangle_{A}\right\}$ and $\left\{|i\rangle_{B}\right\}$ are orthonormal, $d=\operatorname{dim}(B)$ and the $U_{j}$ are unitary. Thus, $j$ can be thought of as a label describing which orthonormal basis is used on Bob's system to encode $i$. For such states, a convexity argument (see Ref. [8]) quickly implies that

$$
I_{c}(\rho) \leq \log d+\max _{\varphi} \frac{1}{n} \sum_{i j}\left|\left\langle\varphi\left|U_{j}\right| i\right\rangle\right|^{2} \log \left|\left\langle\varphi\left|U_{j}\right| i\right\rangle\right|^{2} .
$$

The communication of $j$, which requires $l=\log n$ bits, obviously yields a state $\rho^{\prime}$ for which $I_{c}\left(\rho^{\prime}\right)=\log d+\log n$ so an investigation of the locking properties of $\rho$ will hinge on bounding the second term of Eq. (65). Letting $p_{j i}=\left|\left\langle\varphi\left|U_{j}\right| i\right\rangle\right|^{2}$ and $p_{j}=\left(p_{j 1}, \ldots, p_{j d}\right)$, this second term is equal to $-\frac{1}{n} \sum_{j} H\left(p_{j}\right)$. (Note that $p_{j i}$ and $p_{j}$ are functions of $\varphi$, although we have suppressed the dependence in our notation.) As usual, we will proceed by selecting the operators $U_{j}$ at random using the Haar measure, in which case this average entropy quantity will be provably large. 
Indeed, a now familiar type of calculation (see appendix $\mathbb{B}$, with the substitution $\epsilon \mapsto \epsilon / 2$ ) demonstrates that there is a positive constant $C^{\prime \prime}$ such that

$$
\operatorname{Pr}\left(\inf _{\varphi} \frac{1}{n} \sum_{j=1}^{n} H\left(p_{j}\right) \leq(1-\epsilon / 2) \log d-3\right) \leq\left(\frac{20}{\epsilon}\right)^{2 d} \exp \left(-n\left(\frac{\epsilon d C^{\prime \prime}}{4(\log d)^{2}}-1\right)\right),
$$

provided $\epsilon<2 / 5$ and $d \geq 7$. Choosing $n=(\log d)^{3}$ and $\log d$ to be larger than $\frac{16}{C^{\prime \prime} \epsilon} \log \frac{20}{\epsilon}$ then ensures that the probability is bounded away from 1. It's worth pausing to interpret this statement: it means that there is a choice of $n$ bases that is highly incommensurate with all states $\varphi$, in the sense that averaged over bases, the entropy of the probability distribution induced by measuring any fixed $\varphi$ is almost maximal. Returning to locking, we see that there exists a choice of unitaries such that

$$
I_{c}(\rho) \leq \log d-[(1-\epsilon / 2) \log d-3]=\frac{\epsilon}{2} \log d+3 .
$$

We can then estimate, using the facts that $3 / \log d<\epsilon / 2$ and $d \geq 7$,

$$
\begin{aligned}
& r_{1} \leq \frac{\frac{\epsilon}{2} \log d+3}{\log d+3 \log \log d} \leq \epsilon \\
& r_{2} \leq \frac{3 \log \log d}{(1-\epsilon / 2) \log d} .
\end{aligned}
$$

The general mathematical question we addressed in this section amounts to quantifying the constraints imposed by entropic uncertainty relations [24, 25] on typical observables, an interesting problem in its own right, regardless of its connection to locking classical correlations. As such, and acknowledging that the approximations used here were quite crude, it would be worth developing a more detailed understanding of the distribution of the quantity $\min _{\varphi} \sum_{j=1}^{n} H\left(p_{j}\right)$.

\section{DISCUSSION}

We have explored a range of cryptographic applications that are based on concentration phenomena in high-dimensional inner product spaces. Most results in quantum information theory exploit regularities in the structure of the input or operations; Schumacher's quantum noiseless coding theorem [20, 26], for example, is based on the fact that for large $l$, a state $\rho^{\otimes l}$ will be almost entirely supported on an $l(S(\rho)+\delta)$-qubit subspace. The results we presented here are of a related but different character: the regularity we exploit is inherent in the structure of $\mathbb{C}^{d}$ and, therefore, doesn't require any additional constraints.

Our first application was to demonstrate the existence of approximate private quantum channels capable of achieving exponential security (as measured, for example, by an eavesdropper's accessible information) in the number of encrypted qubits while simultaneously using only about half as much key as the well-known perfectly secure constructions. The failure of bounds on the size of the secret key from the exact case to apply in our approximate setting exposed a new distinction between quantum and classical correlations: classical correlations must be destroyed by local randomization operations while quantum correlations can survive such operations. Our second application built on this principle to find protocols for LOCC hiding of bipartite quantum states capable of encoding roughly $l$ qubits in $2 l$ qubits, a significant improvement over previous constructions. We ended by exhibiting states with locked classical correlations. Such states can be used to perform surprising communication tasks but also serve as a warning that accessible information is a potentially volatile measure for use in security definitions. 
Our results here suggest a number of possible directions for future research. One natural question is the optimality of the cryptographic protocols we've described. While a simple rank argument ensures that the $1: 1$ asymptotic ratio of secret key bits to encoded qubits achieved by our approximate private quantum channel is optimal assuming a unitary encryption map, there appear to be technical obstacles to proving optimality in case of CPTP encryption maps with unbounded output dimension. (If the size of the encrypted state is a polynomial function of the size of the message then the proof is straightforward. One need only combine the argument of Ref. [6], Section IV with the Fannes inequality [27].) Optimality of the $1: 2$ ratio found for quantum data hiding represents an even bigger challenge; we know of no convincing argument, beset by technical obstacles or not. At a finer level of detail, since our focus has been on asymptotic rates, we haven't made any serious attempt to optimize the constants in our constructions; it is likely that significant improvements and perhaps simplifications could be found, particularly in the estimates leading to the locking result. Finally, from a practical point of view, the most pressing problem would be to find computationally efficient versions of the constructions we have presented here. Our Appendix A provides one step in this direction in the case of approximate private quantum channels: instead of selecting unitary transformations from the full unitary group, it suffices to select them at random from the set of products of Pauli operators. This random selection is easily done in polynomial time and the Pauli operators are easily implemented. Unfortunately, since the number of random selections is exponential in the number of encrypted qubits, this simplification does not yet yield a polynomial time construction.

\section{Acknowledgments}

We thank Daniel Gottesman, Leonid Gurvits, Karol Zyczkowski and, in particular, Charles Bennett for their helpful suggestions. PH and DL acknowledge the support of the Sherman Fairchild Foundation, the Richard C. Tolman Foundation, the Croucher Foundation and the US National Science Foundation under grant no. EIA-0086038. AW is supported by the U.K. Engineering and Physical Sciences Research Council.

\section{APPENDIX A: BEYOND HAAR MEASURE}

For some applications, a randomization procedure using a different distribution over unitaries would be preferable; in data hiding, for example, proving that the decoding procedure succeeds with high probability would have been greatly facilitated by using Pauli operators instead of arbitrary unitaries. The convenience appears to come at a price, however. Rather than the strong operator norm estimate of section $\amalg$, we have only been able to prove the corresponding result in trace norm for the general case.

Throughout, we assume only that $\left\{U_{j}: 1 \leq j \leq n\right\}$ are independent $\mathrm{U}(d)$-valued random variables with density $p(U)$ such that $\mathbb{E} U_{j} \varphi U_{j}^{\dagger}=\mathbb{I} / d$. The density function $p(U)$, for example, could consist of point masses concentrated at tensor products of Pauli operators.

Lemma A.1 For a fixed pure state $\varphi, \mathbb{E}\left[\left\|\frac{1}{n} \sum_{j=1}^{n} U_{j} \varphi U_{j}^{\dagger}-\mathbb{I} / d\right\|_{1}\right] \leq \sqrt{d / n}$.

Proof First we evaluate

$$
\mathbb{E}\left[\left\|\frac{1}{n} \sum_{j=1}^{n} U_{j} \varphi U_{j}^{\dagger}-\frac{\mathbb{I}}{d}\right\|_{2}^{2}\right]=\frac{1}{n^{2}} \sum_{i j} \mathbb{E} \operatorname{Tr}\left(U_{i} \varphi U_{i}^{\dagger} U_{j} \varphi U_{j}^{\dagger}\right)-\frac{1}{d} .
$$


The expectation value is easy to calculate:

$$
\begin{aligned}
\sum_{i j} \mathbb{E} \operatorname{Tr}\left(U_{i} \varphi U_{i}^{\dagger} U_{j} \varphi U_{j}^{\dagger}\right) & =\sum_{i} \mathbb{E} \operatorname{Tr}\left(U_{i} \varphi U_{i}^{\dagger}\right)+\sum_{i \neq j} \mathbb{E} \operatorname{Tr}\left(U_{i} \varphi U_{i}^{\dagger} U_{j} \varphi U_{j}^{\dagger}\right) \\
& =n+\sum_{i \neq j} \operatorname{Tr}\left(\frac{\mathbb{I}}{d} \frac{\mathbb{I}}{d}\right) \\
& =n+\frac{n(n-1)}{d} .
\end{aligned}
$$

Therefore, $\mathbb{E}\left[\frac{1}{n}\left\|\sum_{j=1}^{n} U_{j} \varphi U_{j}^{\dagger}-\frac{\mathbb{I}}{d}\right\|_{2}^{2}\right]=\frac{d-1}{n d}$. By the Cauchy-Schwartz inequality and the concavity of the square-root function, we can then estimate

$$
\begin{aligned}
\mathbb{E}\left[\left\|\frac{1}{n} \sum_{j=1}^{n} U_{j} \varphi U_{j}^{\dagger}-\frac{\mathbb{I}}{d}\right\|_{1}\right] & \leq \sqrt{d \mathbb{E}}\left[\left\|\frac{1}{n} \sum_{j=1}^{n} U_{j} \varphi U_{j}^{\dagger}-\frac{\mathbb{I}}{d}\right\|_{2}\right] \\
& \leq \sqrt{d}\left(\mathbb{E}\left[\left\|\frac{1}{n} \sum_{j=1}^{n} U_{j} \varphi U_{j}^{\dagger}-\frac{\mathbb{I}}{d}\right\|_{2}^{2}\right]\right)^{1 / 2} .
\end{aligned}
$$

The lemma then follows by substitution and the trivial inequality $d-1<d$.

We'll also make use of Azuma's inequality:

Lemma A.2 Let $\left(Y_{j}\right)_{j=1}^{n}$ be a sequence of real-valued random variables such that $\left|Y_{j}\right| \leq 1$. Let $S_{n}=$ $\sum_{j=1}^{n} Y_{j}$ and $S_{0}=0$. If $\mathbb{E}\left[Y_{j} \mid S_{j-1}\right]=0$, then

$$
\operatorname{Pr}\left(\frac{1}{n} S_{n} \geq t\right) \leq \exp \left(\frac{-n t^{2}}{2}\right) .
$$

Proof See, for example, Ref. [15].

Theorem A.3 For sufficiently large $d$ and $n=d \log d / \epsilon^{2}$, there exists a choice of $\left\{U_{j}\right\}_{j=1}^{n}$ in the support of $p$ such that the inequality

$$
\left\|\frac{1}{n} \sum_{j=1}^{n} U_{j} \varphi U_{j}^{\dagger}-\frac{\mathbb{I}}{d}\right\|_{1} \leq \epsilon
$$

holds for all states $\varphi$.

Proof For $n, k \geq 1$, let $Z_{n}=\left\|\sum_{j=1}^{n} U_{j} \varphi U_{j}^{\dagger}-n \mathbb{I} / d\right\|_{1}, S_{k}=\mathbb{E}\left[Z_{n} \mid U_{1}, \ldots, U_{k}\right]-\mathbb{E}\left[Z_{n}\right]$ and $Y_{k}=$ $S_{k}-S_{k-1}$. It's also convenient to introduce the notation $S_{0}=Y_{0}=0$. Note that

$$
\mathbb{E}\left[Y_{k} \mid S_{k-1}\right]=\mathbb{E}\left[\mathbb{E}\left[S_{k} \mid U_{1}, \ldots, U_{k-1}\right]-S_{k-1} \mid S_{k-1}\right]=0
$$

Also, for fixed $\left(U_{1}, \ldots, U_{n}\right)$ and unitary $\hat{U}_{k}$, the triangle inequality gives

$$
\begin{aligned}
& \quad\left|\left\|\sum_{j=1}^{n} U_{j} \varphi U_{j}^{\dagger}-\frac{n \mathbb{I}}{d}\right\|_{1}-\left\|\sum_{j \neq k} U_{j} \varphi U_{j}^{\dagger}+\hat{U}_{k} \varphi \hat{U}_{k}^{\dagger}-\frac{n \mathbb{I}}{d}\right\|_{1}\right| \\
& \leq\left\|U_{k} \varphi U_{k}^{\dagger}-\hat{U}_{k} \varphi \hat{U}_{k}^{\dagger}\right\|_{1} \leq 2
\end{aligned}
$$


so $\left|Y_{k}\right| \leq 2$. An application of lemma A.2 to $S_{n}=\sum_{k=1}^{n} Y_{k}$ then tells us that

$$
\operatorname{Pr}\left(Z_{n}-\mathbb{E}\left[Z_{n}\right] \geq 2 n t\right) \leq \exp \left(\frac{-n t^{2}}{2}\right) .
$$

By the previous lemma, if $n \geq 4 d / \delta^{2}$, then $\mathbb{E}\left[Z_{n}\right] \leq n \delta / 2$. Therefore, when this condition holds, we find that

$$
\operatorname{Pr}\left(Z_{n} \geq n \delta\right) \leq \exp \left(\frac{-n \delta^{2}}{32}\right) .
$$

Fix an $\epsilon / 2$-net $\mathcal{M}$ with $|\mathcal{M}| \leq(10 / \epsilon)^{2 d}$. Then

$$
\operatorname{Pr}\left(\sup _{\varphi}\left\|\sum_{j=1}^{n} U_{j} \varphi U_{j}^{\dagger}-\frac{n \mathbb{I}}{d}\right\|_{1} \geq n \epsilon\right) \leq \operatorname{Pr}\left(\max _{\varphi \in M}\left\|\sum_{j=1}^{n} U_{j} \varphi U_{j}^{\dagger}-\frac{n \mathbb{I}}{d}\right\|_{1} \geq \frac{n \epsilon}{2}\right) .
$$

By the union bound and our previous calculations, this probability is less than or equal to

$$
\left(\frac{10}{\epsilon}\right)^{2 d} \exp \left(\frac{-n \epsilon^{2}}{128}\right)
$$

If $n=d \log d / \epsilon^{2}$, then this quantity goes to zero with increasing $d$.

\section{APPENDIX B: PROOF OF EQ. (66)}

Our goal is to prove that there is a positive constant $C^{\prime \prime}$ such that

$$
\operatorname{Pr}\left(\inf _{\varphi} \frac{1}{n} \sum_{j=1}^{n} H\left(p_{j}\right) \leq(1-\epsilon) \log d-3\right) \leq\left(\frac{10}{\epsilon}\right)^{2 d} \exp \left(-n\left(\frac{\epsilon C^{\prime \prime} d}{2(\log d)^{2}}-1\right)\right)
$$

where $p_{j i}=\left|\left\langle i\left|U_{j}^{\dagger}\right| \varphi\right\rangle\right|^{2}$.

We begin by estimating the concentration of measure for the entropy of measurement of a random state. Let $|\psi\rangle$ be a pure state chosen from the unitarily invariant measure on $\mathbb{C}^{d}, q_{i}=$ $|\langle i \mid \psi\rangle|^{2}$ and $f(|\psi\rangle)=H(q)$ the Shannon entropy of the distribution $q$. We use a version of Levy's Lemma [28]:

Lemma B.1 (Levy) Let $f: S^{k-1} \rightarrow \mathbb{R}$ be a function with Lipschitz constant $\sigma$. Then

$$
\operatorname{Pr}(|f-\mathbb{E} f|>\eta) \leq 4 \exp \left(-C^{\prime} k \eta^{2} / \sigma^{2}\right)
$$

for Haar measure on $S^{k-1}$ and $C^{\prime}>1 /(220 \ln 2)$ a constant.

For our application, $k=2 d$ and the Lipschitz constant can be expressed in terms of the $q_{i}$ :

$$
\begin{aligned}
\sigma^{2}=\sup _{\psi} \nabla f \cdot \nabla f & =\frac{4}{(\ln 2)^{2}} \sum_{i=1}^{d} q_{i}\left(1+\ln q_{i}\right)^{2} \\
& \leq \frac{4}{(\ln 2)^{2}} \sum_{i=1}^{d} q_{i}\left(1+\left(\ln q_{i}\right)^{2}\right) \\
& \leq \frac{4}{(\ln 2)^{2}}\left(1+(\ln d)^{2}\right) \leq 8(\log d)^{2}
\end{aligned}
$$


where the second inequality, true if $d \geq 3$, can be shown using Lagrange multipliers. The expectation value of $f$ is $\log d-\Delta(d)$, with $\Delta(d)=\log d-\left(\frac{1}{2}+\frac{1}{3}+\cdots \frac{1}{d}\right) /(\ln 2)$, which converges to $(1-\gamma) /(\ln 2)$, where $\gamma$ is Euler's constant (approximately 0.577) [29]. Using the estimate [30]

$$
\frac{1}{2(d+1)}<\sum_{i=1}^{d} \frac{1}{i}-\ln d-\gamma<\frac{1}{2 d}
$$

we can guarantee that $1 / 2<\Delta(d)<1$ if $d \geq 7$. Thus, choosing $\eta=2-\Delta(d)$ and setting $C^{\prime \prime}=C^{\prime} / 8$, we find

$$
\operatorname{Pr}(H(q)<\log d-2) \leq 4 \exp \left(-\frac{d C^{\prime \prime}}{(\log d)^{2}}\right)
$$

We now move on to bounding $\sum_{j=1}^{n} H\left(p_{j}\right)$ for a given $\varphi$. This is easily done using the Chernoff bound [15]: if $X_{1}, \ldots, X_{n}$ are i.i.d. random variables such that $X_{j} \in[0,1]$ and $\mathbb{E} X=\mu \geq \alpha \geq 0$, then

$$
\operatorname{Pr}\left(\frac{1}{n} \sum_{j=1}^{n} X_{j} \leq \alpha\right) \leq \exp (-n D(\alpha \| \mu))
$$

where $D(\cdot \| \cdot)$ is the binary divergence function

$$
D(\alpha \| \mu)=\alpha \log \alpha-\alpha \log \mu+(1-\alpha) \log (1-\alpha)-(1-\alpha) \log (1-\mu) .
$$

Let $X_{j}=0$ whenever $H\left(p_{j}\right)<\log d-2$ and $X_{j}=1$ otherwise. Then $(\log d-2) X_{j} \leq H\left(p_{j}\right)$ and by Eq. (B6), $\mathbb{E}_{U} X_{j} \geq 1-4 \exp \left(-d C^{\prime \prime} /(\log d)^{2}\right)$. Choosing $\alpha=1-\frac{\epsilon}{2}$ and $\mu=\mathbb{E}_{U} X_{j}$ in the Chernoff bound, we find

$$
\operatorname{Pr}\left(\frac{1}{n} \sum_{j=1}^{n} H\left(p_{j}\right) \leq\left(1-\frac{\epsilon}{2}\right)(\log d-2)\right) \leq \exp \left(-n D\left(1-\frac{\epsilon}{2} \| 1-4 \exp \left(\frac{-d C^{\prime \prime}}{(\log d)^{2}}\right)\right) .\right.
$$

The divergence can be bounded as follows:

$$
\begin{aligned}
D\left(1-\frac{\epsilon}{2} \| 1-4 \exp \left(\frac{-d C^{\prime \prime}}{(\log d)^{2}}\right)\right) & \geq-H(\epsilon / 2)-\epsilon+\frac{\epsilon d C^{\prime \prime}}{2(\log d)^{2}} \\
& \geq \frac{\epsilon d C^{\prime \prime}}{2(\log d)^{2}}-1 .
\end{aligned}
$$

The first inequality arises by neglecting the mixed term corresponding to $\alpha \log \mu$ in the divergence, since it is always nonnegative. The second is valid whenever $\epsilon<1 / 5$, which we assume from now on.

To extend Eq. (B9) to all possible states, choose an $\frac{\epsilon}{2}$-net $\mathcal{M}$ for $d$-dimensional pure states, with $|\mathcal{M}|=(10 / \epsilon)^{2 d}$. Write $\tilde{\varphi}$ for the net point corresponding to $\varphi$. Let $p_{j}$ be as previously defined in terms of $\varphi$, and $\tilde{p_{j}}$ be similarly defined in terms of $\tilde{\varphi}$. Using the union bound,

$$
\operatorname{Pr}\left(\min _{\tilde{\varphi} \in \mathcal{M}} \frac{1}{n} \sum_{j=1}^{n} H\left(\tilde{p_{j}}\right) \leq\left(1-\frac{\epsilon}{2}\right)(\log d-2)\right) \leq|\mathcal{M}| \exp \left[-n\left(\frac{\epsilon d C^{\prime \prime}}{2(\log d)^{2}}-1\right)\right] .
$$

Furthermore, viewing $p_{j}$ and $\tilde{p}_{j}$ as postmeasurement states, the monotonicity of the trace norm implies

$$
\left\|p_{j}-\tilde{p}_{j}\right\|_{1} \leq \frac{\epsilon}{2}
$$


Then, applying Fannes' inequality [27] to the distributions $p_{j}$ and $\tilde{p}_{j}$,

$$
\left|H\left(p_{j}\right)-H\left(\tilde{p_{j}}\right)\right| \leq \frac{\epsilon}{2} \log d-\frac{\epsilon}{2} \log \frac{\epsilon}{2} \leq \frac{\epsilon}{2} \log d+1
$$

A substitution then completes the proof.

[1] S. Braunstein, H.-K. Lo, and T. Spiller. Forgetting qubits is hot to do. Unpublished manuscript, 1999.

[2] P. O. Boykin and V. Roychowdhury. Optimal encryption of quantum bits. arXiv quant-ph/0003059.

[3] A. Ambainis, M. Mosca, A. Tapp, and R. de Wolf. Private quantum channels. In IEEE Symposium on Foundations of Computer Science (FOCS), pages 547-553, 2000. arXiv quant-ph/0003101.

[4] C. H. Bennett and S. Wiesner. Communication via one- and two-particle operators on EinsteinPodolsky-Rosen states. Phys. Rev. Lett., 69(20):2881-2884, 1992.

[5] T. Eggeling and R. F. Werner. Hiding classical data in multi-partite quantum states. Phys. Rev. Lett., 89(9):097905, 2002. arXiv quant-ph/0203004.

[6] D. P. DiVincenzo, P. Hayden, and B. M. Terhal. Hiding quantum data. Found. Phys., 33(11):1629-1647, 2003. arXiv quant-ph/0207147.

[7] D.P. DiVincenzo, D. W. Leung, and B. M. Terhal. Quantum data hiding. IEEE Trans. Inf. Theory, 48(3):580-598, 2002. arXiv quant-ph/0103098.

[8] D. P. DiVincenzo, M. Horodecki, D. Leung, J. Smolin, and B. M. Terhal. Locking classical correlation in quantum states. arXiv quant-ph/0303088.

[9] C. H. Bennett, P. Hayden, D. Leung, P. W. Shor, and A. Winter. Remote preparation of quantum states. arXiv quant-ph/0307100.

[10] C. H. Bennett, G. Brassard, C. Crépeau, R. Jozsa, A. Peres, and W. K. Wootters. Teleporting an unknown quantum state via dual classical and Einstein-Podolsky-Rosen channels. Phys. Rev. Lett., 70:1895-1899, 1993.

[11] H.-K. Lo. Classical-communication cost in distributed quantum-information processing: A generalization of quantum-communication complexity. Phys. Rev. A, 62:012313, 2000.

[12] D. Leung. Quantum vernam cipher. Quantum Info. Comp., 2:14-34, 2001.

[13] D. Leung and P. Shor. Oblivious remote state preparation. Phys. Rev. Lett., 90:127905, 2003.

[14] A. S. Holevo. Statistical problems in quantum physics. In G. Maruyama and J. V. Prokhorov, editors, Proceedings of the second Japan-USSR Symposium on Probability Theory, volume 330 of Lecture Notes in Mathematics, pages 104-119, Berlin, 1973. Springer-Verlag.

[15] A. Dembo and O. Zeitouni. Large deviations techniques and applications. Springer-Verlag, New York, 1993.

[16] K. Zyczkowski and H.-J. Sommers. Truncations of random unitary matrices. J. Phys. A, 33:2045-2057, 2000.

[17] C. H. Bennett, D. P. DiVincenzo, C. A. Fuchs, T. Mor, E. Rains, P. W. Shor, J. A. Smolin, and W. K. Wootters. Quantum nonlocality without entanglement. Phys. Rev. A, 59(2):1070-1091, 1999. arXiv quant-ph/9804053.

[18] P. Hayden. Spin-cycle entanglement. In preparation.

[19] F. Verstraete and J. I. Cirac. Quantum nonlocality in the presence of superselection rules and some applications. arXiv quant-ph/0302039.

[20] M. Ohya and D. Petz. Quantum entropy and its use. Texts and monographs in physics. Springer-Verlag, Berlin, 1993.

[21] P. Hausladen and W. K. Wootters. A pretty good measurement for distinguishing quantum states. J. Mod. Opt., 41:2385-2390, 1994.

[22] P. Hausladen, R. Jozsa, B. Schumacher, M. Westmoreland, and W. K. Wootters. Classical information capacity of a quantum channel. Phys. Rev. A, 54:1869-1876, 1996.

[23] C. A. Fuchs and J. van de Graaf. Cryptographic distinguishability measures for quantum mechanical states. IEEE Trans. Inf. Theory, 45:1216-1227, 1999. arXiv quant-ph/9712042.

[24] D. Deutsch. Uncertainty in quantum measurements. Phys. Rev. Lett., 50:631-633, 1983.

[25] H. Maasen and I. Uffink. Generalized entropic uncertainty relations. Phys. Rev. Lett., 60:1103-1106, 1988. 
[26] B. Schumacher. Quantum coding. Phys. Rev. A, 51:2738-2747, 1995.

[27] M. Fannes. A continuity property of the entropy density for spin lattice systems. Comm. Math. Phys., 31:291-294, 1973.

[28] V.D. Milman and G. Schechtman. Asymptotic theory of finite dimensional normed spaces. Number 1200 in Lecture Notes in Mathematics. Springer-Verlag, 1986.

[29] R. Jozsa, D. Robb, and W.K. Wootters. Lower bound for accessible information in quantum mechanics. Phys. Rev. A, 49(2):668-677, 1994.

[30] R. M. Young. Euler's constant. Math. Gaz., 75:187-190, 1991. 